\title{
Simultaneous Imaging of PhytoPlankton AND ZOOPLANKTON DISTRIBUTIONS
}

\author{
By Jules S. Jaffe, Peter J.S. Franks \\ and Andrew W. Leising
}

$\mathrm{U}$ NDERSTANDING THE DYNAMICS of the relationship between phytoplankton and higher trophic levels is necessary for understanding the transfers of matter and energy through planktonic food webs. There have been several suggestions in the literature that copepods were more strongly associated with the subsurface productivity maximum than the chlorophyll maximum (Herman, 1983; Herman and Platt, 1983; Napp et al., 1988). Zooplankton have been shown to respond to microscale (centimeters) patches of phytoplankton in the lab (Tiselius, 1992; Saiz et al., 1993), however, except for Tiselius et al. (1994), who found correlations to $15 \mathrm{~cm}$, there have been few data to confirm these results in situ. Zooplankton behavioral response to their food environment is therefore a critical factor to consider in trying to predict the relationship between phytoplankton and zooplankton in the ocean.

Testing of hypotheses regarding zooplankton-phytoplankton associations in situ has been difficult due to problems associated with sampling resolution. Traditional net sampling methods integrate over the small ( $\mathrm{cm}$ to $\mathrm{m}$ ) spatial scales relevant to exploring such relationships. Phytoplankton sampling by bottles or fluorometers generally gives spatial resolutions no finer than tens of centimeters (Bjorenson and Nielsen, 1991). Recent developments of in situ fluorometers have allowed observation of vertical variability to several centimeter resolution (Derenbach et al., 1979: Cowles and Desiderio, 1993: Cowles et al., 1993). Unfortu-

Jules S. Jaffe, Peter J.S. Franks and Andrew W. Leising. Scripps Institution of Oceanography, La Jolla, CA 92093-0238. USA. nately, similar resolution of zooplanktonic fields using noninvasive sampling techniques has been lacking due to technological constraints.

The high-resolution spectral fluorometer of Cowles (e.g., Cowles and Desiderio, 1993) has allowed observations of the microscale structure of both chlorophyll and phycoerythrin fluorescence (Cowles et al., 1993). Both chlorophyll and phycoerythrin were seen to have significant variability at tens of centimeters, but the two fields were quite uncorrelated with each other. These observations raise many important questions concerning physical-biological couplings in the plankton at small scales and zooplankton foraging behavior in a patchy environment. For example, what physical and biological processes might be operating to allow formation and persistence of such thin layers or small patches of high phytoplankton biomass? What are the time and space scales of persistence of such features? Are the scales of phytoplankton patchiness the same for different taxa? And, in particular for this study, are the patches available to, or exploited by the zooplankton? The answers to such questions may alter our ideas about patchiness, production, and trophodynamics in the ocean.

To attempt to answer some of these questions, two instruments were developed: FishTV (Jaffe et al.. 1995) and OSST (Optical Serial Section Tomography) (Palowitch and Jaffe, 1994, 1995). FishTV uses ultrasound $(445 \mathrm{kHz})$ to locate micronekton $(>1 \mathrm{~cm})$ in a three-dimensional (3D) volume. OSST measures laser-stimulated fluorescence over a $70 \times$ $70 \mathrm{~cm}$ sheet with $0.67-\mathrm{cm}$ resolution. These instruments were profiled simultaneously, imaging the same water 30 miles west of San Diego in the southern California Bight. Here we focus on the relationship between the acoustic and optical depth profiles.

\section{Methodology}

\section{FishTV}

FishTV consists of a set of eight projecting transducers and eight receiving transducers that operate at a frequency of $445 \mathrm{kHz}$. Together, the transmitters and receivers form an $8 \times 8$ image with a field of view of $16 \times 16^{\circ}$, at frame rates up to $4 \mathrm{~Hz}$. Data reported in this article were obtained with the system operating at a frame rate of $2 \mathrm{~Hz}$. Range resolution is $\sim 2.5 \mathrm{~cm}$. In a typical deployment mode, the system images a volume that starts at a range of $2.5 \mathrm{~m}$ and ends at 6.3 $\mathrm{m}$ range, giving a volume of $\sim 6 \mathrm{~m}^{3}$. Individual locations of targets (echo counting) can then be computed accurately if densities are $<40-50 \mathrm{~m}^{-3}$ (see below) (Jaffe et al.. 1995). The system has recently been used to track several hundred targets and infer properties about the trajectories of the animals (McGehee and Jaffe, 1996).

The sonar imaging system is calibrated over its entire field of view and therefore provides values of target strengths as a function of position. To register the presence of an animal, a threshold signal-tonoise ratio of the signal was used. If a reflection exceeded this threshold for several range cells (a sonar pulse extends for several range cells), an animal was judged to be present, and its position in 3D was recorded. Because the beam patterns of the system yield large readings in the area surrounding a target, even though no animals may be present, these regions were excluded from further analysis when a target was detected. Monte Carlo simu- 
lations of the ability of the system to correctly echo-count uniformly distributed targets indicate that this effect will have no significant influence on the estimated number of targets, up to densities of $40-50 \mathrm{~m}^{-3}$. At higher densities the system will undercount the number of individuals. A frame consists of the 3D positions and target strength of the set of animals that were in the field of view of the sonar system over a system cycle. Frames were indexed as a function of depth, so that the optical and sonar recordings could be cross-referenced. FishTV acquires images by pulsing a transmitting array sequentially. In this deployment an entire image was collected in $0.14 \mathrm{~s}$.

Several issues need to be addressed in interpreting the sonar images and, in particular, assessing whether our estimates of animal densities are absolutely accurate. The short answer is that they are not; they are most certainly an underestimate of abundance. However, if the composition of the animal population does not change appreciably over the water depth, the relative abundance as a function of depth will be accurate. For example, Stanton et al. (1996) have shown in a recent survey of Georges Bank that taxa of similar sizes at the site had large differences in backscatter (e.g., gastropods versus crustaceans). This led them to conclude that they could not equate volumetric backscatter strength with biomass in a simple way. In our case, vertical net tows showed no evidence of gastropods or similar animals. Crustacean zooplankton were the primary source of acoustic variability. In addition, Deamer and Martin (1995) have shown that in using a narrow band sonar system, such as FishTV, care must be taken in equating the target strength measurements

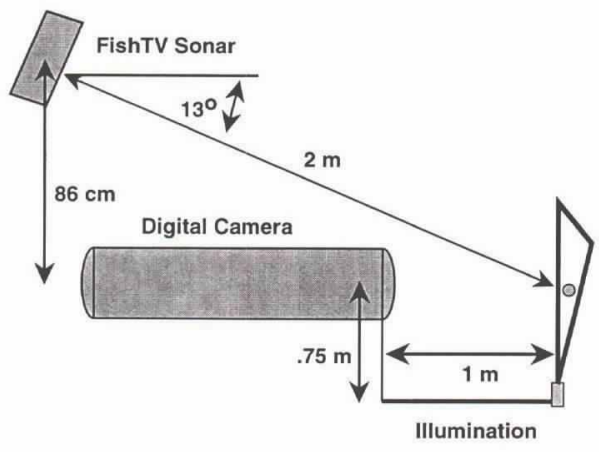

Fig. 1: A schematic diagram of the FishTV and OSST systems showing the geometric placement of the instruments.

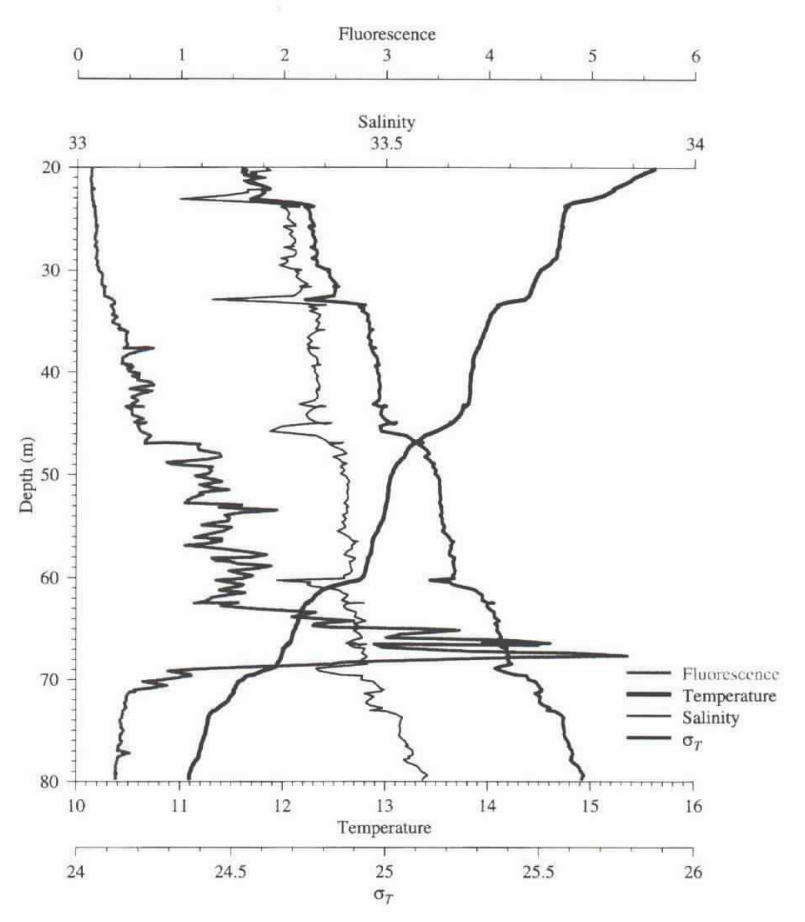

Fig. 2: A CTD/fluorometer profile taken $1 \mathrm{~h}$ before the simultaneous optical and sonar profiles were collected.

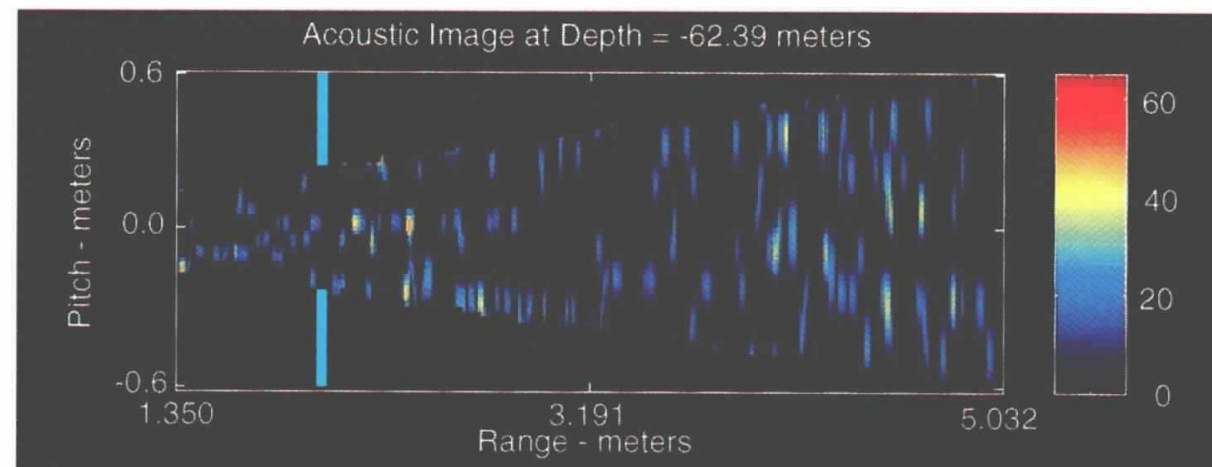

Optical Image Fluorescence \& Abundance vs Depth

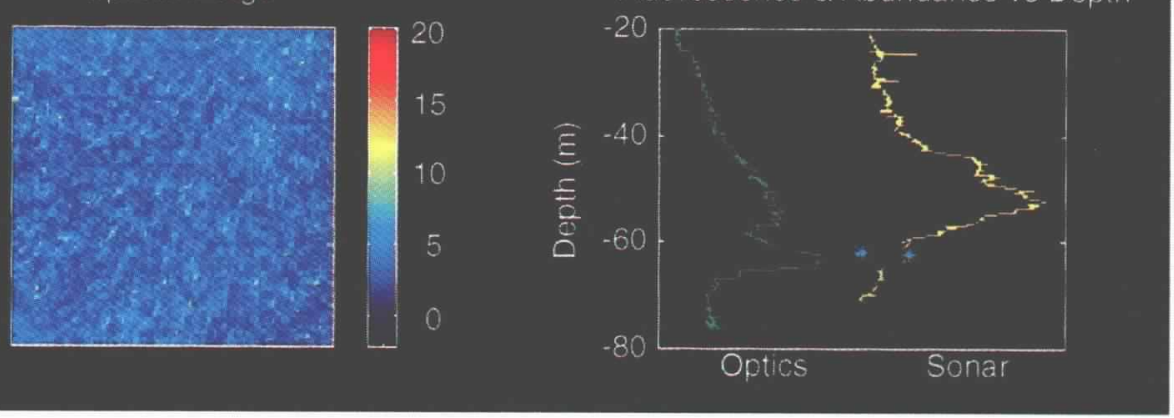

Fig. 3: Simultaneous display of sonar and optical raw data at $60 \mathrm{~m}$ depth: (a) a 2D image (a side-view projection) of the sonar data, (relative) target strengths of the objects are shown on the left-hand side of the frame, (b) one frame of optical data, and (c) simultaneous vertical profiles of the optical and acoustical data sets showing the location of the current frame (asterisk). Raw acoustic images were corrected for range spreading and unequal gains among transmitterreceiver pairs. 
with animal size. This is because the backscatter pattern (frequency- and angledependent target strength) of the animals have 20-30 dB nulls that can lead to serious underestimation of animal size. Another complication is the animal orientation: animals viewed from the side reflect much more sound than those viewed head-on. Finally, our current algorithm does not perform subvoxel (voxel $=3 \mathrm{D}$ pixel) positional estimation, which leads to an underestimate of target strength. All of these effects tend to bias our results toward an underestimation of both animal abundance and target strengths. However, if animal orientations are not a function of depth, and the species composition is constant, our estimates are a good measure of relative abundance. Because species composition is likely to change as a function of depth, the target strength estimates that we derive cannot be correlated with animal size. On the other hand, the estimates of animal abundance will be valid.

Multibeam sonar systems such as FishTV allow finer resolution, or access to more resolution cells (in a given signal-to-noise level) than is possible using conventional technologies. Dual-beam systems do not allow 3D localization of acoustic targets, and both dual-beam and split-beam systems saturate at much lower animal densities than the multibeam system. This may preclude their use in many oceanic situations.

The acoustic data from a multibeam system yield information about both animal abundance and their locations through echo counting (counting of individual targets). At high animal densities, all acoustic systems will be unable to distinguish individual targets. At these densities, systems will switch from echo counting to echo integration, and no resolution of individual targets is possible. Echo counting is widely regarded as the most accurate measure of animal abundances (Stanton and Clay, 1986).

\section{OSST}

Data included in this manuscript represent the first at-sea results with the new optical imaging system. The optical system (OSST) measures laser-induced fluorescence by creating a sheet of illumination and photographing it with a very sensitive $\left(10^{-5}\right.$ lux $)$ charge-coupled device (CCD) camera. Briefly, all lines below $520 \mathrm{~nm}$ of a ship-board argon ion laser were used for stimulation. The fluoresced light was then imaged with a CCD camera
(Photometrics, Tuscon AZ) with a long pass $(>680 \mathrm{~nm})$ filter on the lens. The laser sheet was positioned $\sim 1 \mathrm{~m}$ in front of the camera/sonar package and aligned so that the plane of the image was parallel to the camera plane (Fig. 1). The field of view of the system was $70 \mathrm{~cm} \times 70 \mathrm{~cm}$ over $102 \times 102$ pixels with a resolution of $0.67 \mathrm{~cm}$ and a frame rate of $0.9 \mathrm{~Hz}$.

\section{Combining the Instruments}

The $R / V$ Sproul was moored 30 miles west of San Diego in $300 \mathrm{~m}$ of water on 25-28 July. On the evening of 27 July, numerous vertical profiles were obtained by deploying both the FishTV and OSST systems, as well as temperature probes and a depth sensor. CTD profiles were also performed using a Sea Bird SBE19 CTD interfaced with a Wet Labs "Wet Star" fluorometer and a SeaTech 25-cm path length transmissometer. The instrument package consisting of OSST,

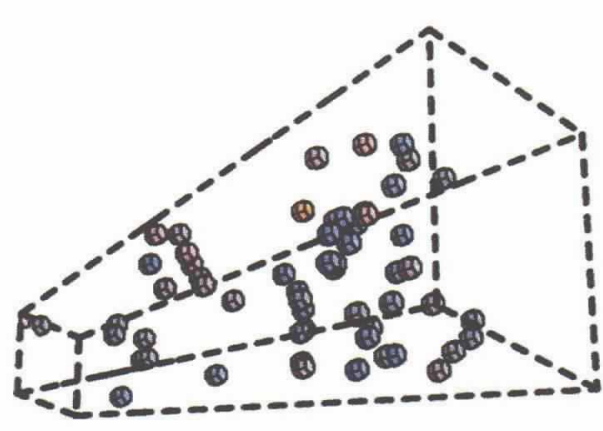

(a)

(a) depth $=31$ meters
FishTV, and depth and temperature sensors was used to profile between 20 and $80 \mathrm{~m}$. In Figures 2 and 3 addition, profiles with the CTD/fluorometer/transmissometer package were made on an hourly basis. In this article, the analysis from a set of four profiles made at 15-min intervals between 2040 and 2140 PST is presented. We estimate that registration between the sonar images and the optical images to $1 \mathrm{~m}$ was achieved, primarily limited by timing accuracies between the various computers that were collecting the information (future versions will resolve this issue).

The FishTV and OSST systems were both mounted on an aluminum frame that was profiled through the water column. Figure 1 shows the geometric configuration of the system that permitted the simultaneous acquisition of both the FishTV and OSST images. Of special note is that the small arm that generates

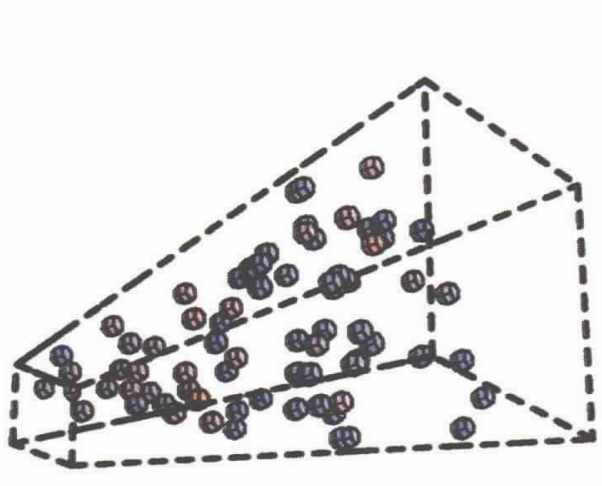

(c) depth $=64$ meters

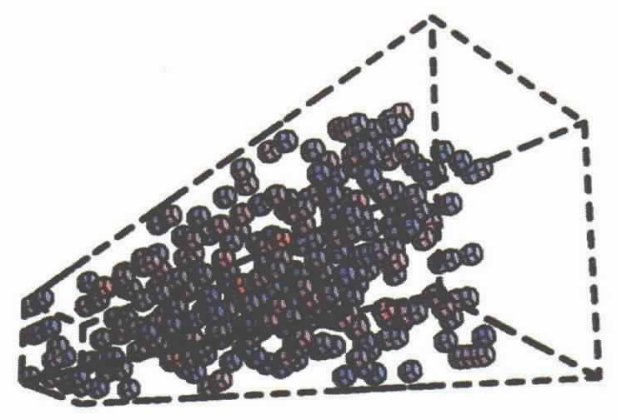

depth $=53$ meters

(b)
Fig. 4: Processed 3D locations of targets at depths of (a) $31 \mathrm{~m}$, (b) $53 \mathrm{~m},(\boldsymbol{c}) 64 \mathrm{~m}$, and (d) $70 \mathrm{~m}$. The dashed line shows the volume imaged by the sonar, starting at $2.0 \mathrm{~m}$ and ending at $5.8 \mathrm{~m}$. Targets varied between (a) $-75 d B$ (red) and $-85 d B$ (blue), (b) -67 $d B$ (red) and $-83 d B$ (blue), (c) $-76 d B$ (red) and $-83 d B$ (blue), and (d) $-75 d B$ (red) and $-83 d B$ (blue). 
the laser sheet is in the acoustic shadow of the digital camera (with respect to the FishTV system). This configuration permitted an exact overlap of both the optical and acoustic images at a range of $2 \mathrm{~m}$ from the acoustic transducers and $\sim 1 \mathrm{~m}$ away from the front of the digital camera case. The sonar was set so that targets starting at a range of $1.8 \mathrm{~m}$ away from the front of the sonar and ending $5.15 \mathrm{~m}$ away were imaged. This yielded a volume of $\sim 3.4 \mathrm{~m}^{3}$ for the system.

\section{Results}

Data from the OSST and FishTV instruments were merged to produce profiles of chl-a fluorescence and animal abundance as a function of depth (Fig. 3). Optical data were averaged over each frame and interpolated onto $10 \mathrm{~cm}$ vertically spaced locations. The acoustic signal was binned by target strength; the signal plotted is the total number of hundreds of targets per $3.4 \mathrm{~m}^{3}$ with acoustic backscatter intensity greater than $-85 \mathrm{~dB}$ (the smallest size class of targets; $\sim 1 \mathrm{~cm}$ ). All such acoustic data are plotted from each profile.

Vertical profiles made with the OSST and the CTD-fluorometer-transmissometer package showed similar small-scale (meters) features: a temporally persistent subsurface chlorophyll maximum layer at $\sim 50 \mathrm{~m}$ (primary maximum), and a sharp ( $<5 \mathrm{~m}$ thick) transient peak centered around $60 \mathrm{~m}$ (secondary maximum; Fig. 3c). This secondary maximum was visible for $\sim 8 \mathrm{~h}$; calculations based on phytoplanktonic growth rates and grazing rates suggest that it was a patch that advected past our anchor site, rather than a feature that formed and dissipated in situ during that time. Profiles of acoustic targets showed a strong correlation with the fluorescence profiles with one major difference: there were almost no animals in the secondary (high chlorophyll, high variance) chlorophyll maximum. The numbers of animals peaked in the primary (lower chlorophyll, lower variance) maximum and decreased rapidly below.

The location of the individual targets at four depths (above the primary maximum, within the primary maximum, in the secondary maximum, and below the secondary maximum) reflect the relative absence of zooplankton from the secondary maximum (Fig. 4). These images can be compared with the corresponding optical images (Fig.

5), which show isotropic, random distributions of fluorescence down to scales of $\sim 3 \mathrm{~cm}$, with less variance than random at smaller scales. Although every effort was made to account for effects of the instrument package motion on these optical images, we cannot reject the possibility that they are an artifact of mixing by the instrument package or poor focus of the camera system. We feel it is likely, however, that these images rep-

$31.86 \mathrm{~m}$

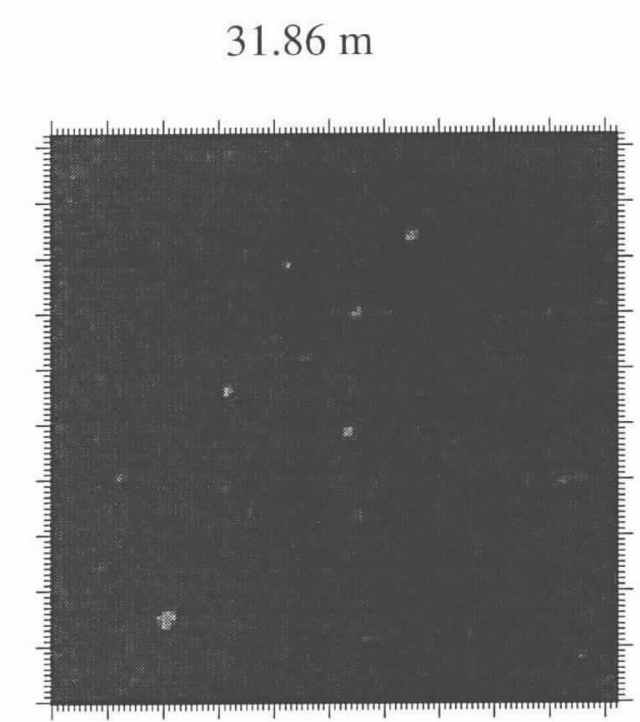

$64.72 \mathrm{~m}$ resent the true, undisturbed state of the fluorescence microstructure, suggesting a very inhomogeneous distribution of fluorescence on microscales.

The mismatch between the fluorescence and acoustic signal is made obvious in a comparison of the four vertical profiles (Fig. 6). This mismatch is persistent over the sampling period $(1 \mathrm{~h})$. Target strengths in the secondary maximum are about equal to background levels (outside the fluorescence maxima). The

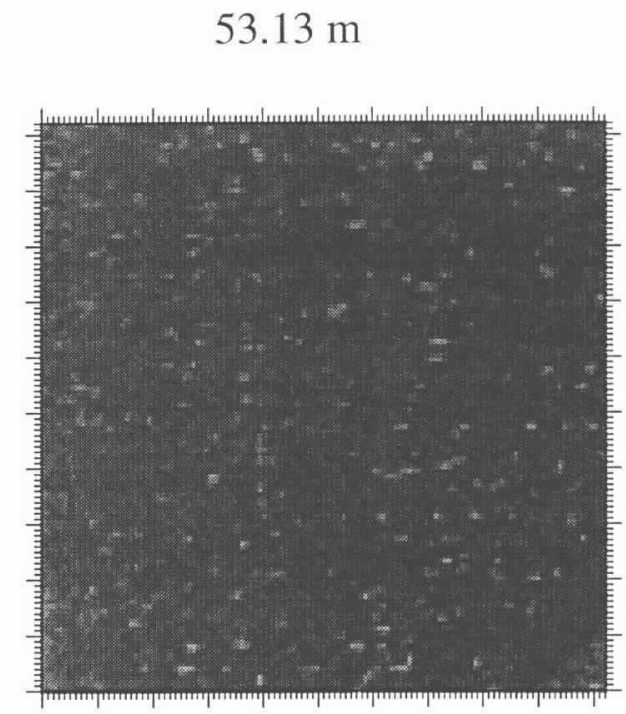

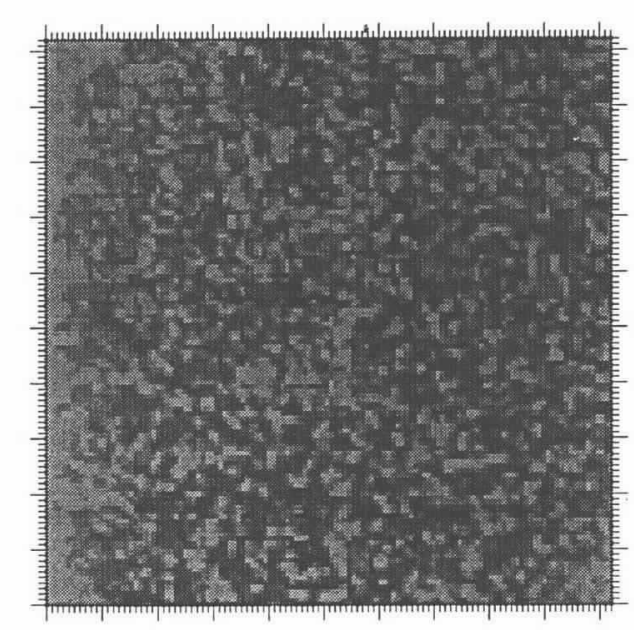

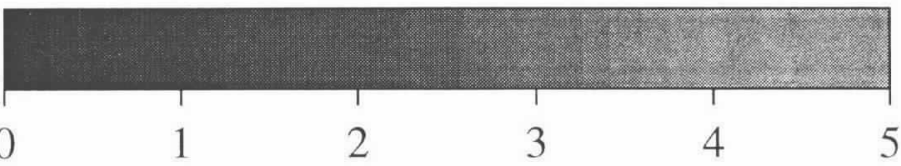

Fig. 5: Four images from the OSST, uncontaminated by ship heave. The images represent the different regions of the water column: $31.86 \mathrm{~m}$ is above the primary fluorescence maximum, 53.13 is in the primary fluorescence maximum, 64.72 is in the secondary maximum, and 68.43 is below the secondary maximum (see profiles in Fig. 2). 

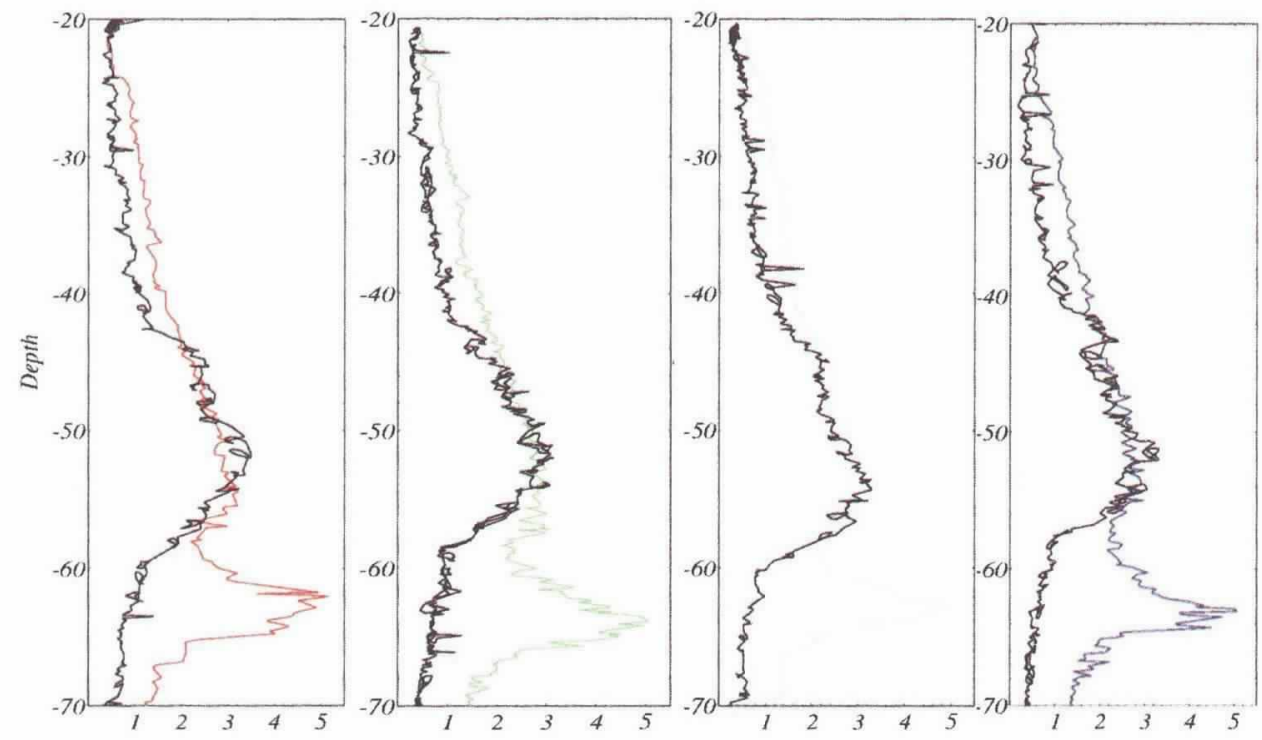

Fig. 6: A comparison of the four optical and acoustical depth profiles. The ordinate shows units in hundreds of animals $/ 3.4 \mathrm{~m}^{3}$ for the acoustic data (in black) and units of relative fluorescence for the optical data (colored).

strong correspondence of the acoustic signal with the primary maximum is obvious in Figure 6. This is the relationship that would be predicted from a first-order model relating chlorophyll and herbivorous zooplankton.

Of the many points to consider in interpreting these data, perhaps the most important one is the effect of ship heave. Other analyses (not shown) demonstrated that small-scale observations were unaffected by the ship motions at the crest and trough of a heave cycle (4-5-s period). In addition, images where ship heave was prominent have average fluorescence values consistent with the fluorescence profile. In the case of the acoustics, because the time it took to collect an entire image was $0.14 \mathrm{~s}$, and because the ship heave values were predominantly $<50 \mathrm{~cm} \mathrm{~s}^{-1}$ (98\% of all values were $<48 \mathrm{~cm} \mathrm{~s}^{-1}$ ), the vertical translation of the platform was typically around 7 $\mathrm{cm}$ during the complete acquisition of a sonar image. In addition, because the image is raster scanned, the delay between adjacent beams is less than this amount. Because the beam widths of the system are only slightly more than this $(6.3 \mathrm{~cm})$ at $1.8-\mathrm{m}$ range and substantially more $(18 \mathrm{~cm})$ at $5.15-\mathrm{m}$ range (the furthest part of the field of view) the translation of the platform had a minor effect on the spatial patterns recorded by the FishTV.

A CTD profile taken $\sim 1 \mathrm{~h}$ before the FishTV profiles (Fig. 2) shows a fairly linear density profile with depth. The chlorophyll peaks at 50 and $65 \mathrm{~m}$ are each flanked by sharp gradients in temperature. These layers represent regions of high temperature gradient formed by straining of the temperature field by internal waves. The high horizontal velocities seen below $55 \mathrm{~m}$ (Franks and Jaffe, unpublished data) suggest that the secondary chlorophyll maximum was advecting horizontally much faster than the primary maximum $\left(20-40 \mathrm{~cm} \mathrm{~s}^{-1}\right.$ versus 10-20 $\mathrm{cm} \mathrm{s}^{-1}$ ).

In addition to the sonar, optical, and CTD profiles, vertical net tows were performed with a single $50-\mathrm{cm}$ diameter Bongo net with a $505-\mu \mathrm{m}$ mesh. The results from a set of vertical hauls to various depths indicate that there was a layer of animals between 50 and $70 \mathrm{~m}$. Estimates of the number of animals in the $1-10-\mathrm{mm}$ size class were 40-50 $\mathrm{m}^{-3}$. Not surprisingly, the number of animals in the $1-\mathrm{cm}$ and above class was very small due to net avoidance. The animal densities recorded by the system at the peak of acoustic reverberation were extremely high, and it is very likely that the system was undercounting the number of animals at this depth $(54 \mathrm{~m})$. Because of a lack of corroborating data, it is impossible to tell exactly what kind of animals were responsible for this peak of acoustic reflectivity; however, it does seem reasonable to assume that they were some type of micronekton.

\section{Discussion}

The data described in this paper represent the first at-sea results from the coupled acoustic/optical system. These data revealed surprising relationships between the small-scale distributions of phytoplankton and zooplankton. The phytoplankton showed a large amount of microscale $(1-10 \mathrm{~cm})$ heterogeneity. The zooplankton showed strong correlations with the "background" fluorescence (i.e., the primary, persistent subsurface fluorescence maximum) but did not show any corresponding enhancement in the most intense fluorescence peak.

One possible explanation for the high number of animal counts in the sonar images was that there was an extremely high number of smaller crustacean zooplankton, such as copepods, and that the sonar was recording a signal that was a superposition of echoes from this large number of animals. This hypothesis can be rejected by noting that the target strengths and densities of the smaller animals are not high enough to lead to the echo-intensities seen at $445 \mathrm{kHz}$. On the other hand, we cannot definitely say that only sonar reflections from micronekton were recorded, because other animals could have been present in the water column that were not sampled by our vertical net tows.

There are more questions created than answered by this first coincident, in situ view of fluorescence and acoustic targets in the ocean. Some of the most compelling directions for future research include measurements of smaller acoustic targets (i.e., higher frequency transducers), auxiliary sampling to test hypotheses about food quality and type, and a better understanding of the response of herbivorous zooplankton to microscale patchiness of their environment. Concurrent use of both optical and sonar imaging systems offers many potential advantages not only for in situ measurement of the spatial distribution and activity level of planktonic organisms but also for the "ground-truthing" of sonar systems. In a recent study, the OASIS system (Jaffe et al., unpublished data) for mapping animals with sonar and simultaneous optical images of them was deployed in Saanich inlet and produced a set of data that yielded target strength information with concurrent optical animal identification. Hopefully the technology presented in this article and additional work by our 
group and others will allow increased understanding of the behavioral responses of zooplankton to their environment and the importance of such dynamics in ensuring the transfer of matter and energy through the food web.

\section{Acknowledgements}

This project would not have been successful without the help of many dedicated people. Particular thanks are due to Greg Adelman and Ed Reuss, who did the bulk of the engineering on the OSST and FishTV systems. Mary Sisti cheerfully organized much of the cruise. Capt. Louis Zimm artfully piloted the $R / V R$. G. Sproul, and Tammi Koonce, Bob Wilson, and Fred Uhlmann were indispensable help in deploying the system. Alex DeRobertis and Adrienne Huston were a great help in sampling, and Dave Zawada processed much of the optical data. Thanks particularly to Eric Shulenberger, Ron Tipper, Steve Ackleson, and Jeff Simmen of the Office of Naval Research for encouraging this enterprise.

\section{References}

Bjorenson P. K. and J. G. Nielsen, 1991: Decimeter scale heterogeneity in the plankton during a pycnocline bloom of Gyrodinium aureolum. Mar, Ecol. Prog. Ser. 73, 263-267.

Cowles. T.J. and R.A. Desiderio, 1993: Resolution of biological microstructure through in-situ fluorescence emission spectra. Oceunography, 6, 105-111.

R.A. Desiderio and S. Neuer, 1993: In situ characterization of phytoplankton from vertical profiles of fluorescence emission spectra. Mar. Biol., 1/5. 217-222.

Deamer. D. and L. Martin, 1995: Zooplankton target strength: volumetric or areal dependence? J. Acoust. Soc. Am.. 98, 1111-1118.

Derenbach. J.B.. H. Astheimer, H.P. Hansen and H. Leach, 1979: Vertical microscale distribution of phytoplankton in relation to the thermocline. Mar. Ecol. Prog. Ser., 1, 187-193.

Herman, A.W., 1983: Vertical distribution patterns of copepods. chlorophyll, and production in north-eastern Baffin Bay. Limnol. Oceanogr., 28, 709-719.

, and T. Platt. 1983: Numerical modeling of diel carbon production and zooplankton grazing on the Scotian shelf based on observational data. Ecol. Modeling, $18,55-72$.

Jaffe, J.S., M.D. Ohman and A. De Robertis, OASIS in the sea: Measurement of the acoustic reflectivity of zooplankton with concurrent optical imaging. Deep-Sea Res. In press.

Jaffe, J.S., E. Reuss, D. McGehee and G. Chandran. 1995: FTV. a sonar for tracking macrozooplankton in 3-dimensions. Deep-Sea Res. 42. 1495-1512.

McGehee, D. and J.S. Jaffe, 1996: Three-dimen- sional swimming behavior of individual zooplankters: observations using the acoustical imaging system FishTV. ICES J. Mar. Sci.. 53, 363-369

Napp, J.M., E.R. Brooks, P. Matrai and M.M. Mullin, 1988: Vertical distribution of marine particles and grazers. II. Relation of grazer distribution to food quality and quantity. Mar. Ecol. Prog. Ser., 50, 59-72.

Palowitch, A.W. and J.S. Jaffe, 1994: Three-dimensional ocean chlorophyll distributions from underwater serial-sectioned fluorescence images. Appl. Opt.. 33, 3023-3033.

and J.S. Jaffe, 1995: Optical serial sectioned chlorophyl-a microstructure. J. Gersphys. Res., 100, 13,267-13,278

Saiz, E.. P. Tiselius, P.R. Johnson. P. Verity and G.-A. Paffenhofer, 1993: Experimental records of the effects of food patchiness and predation of egg production of Acartia tonsa. Limnol. Oceangr., 38, 280-289.

Stanton. T.S.. D. Chu and P.H. Wiebe. 1996: Acoustic scattering characteristics of several zooplankton groups. ICES J. Mar. Sci., 53. 289-295.

and C.S. Clay, 1986: Sonar echo statistics as a remote-sensing tool: volume and seafloor. IEEE J. Oceanic Eng., OE-11, 1, 79--96.

Tiselius. P., 1992: Behavior of Acartia tonsa in patchy food environments. Limnol. Oceanogr. 37. 1640-1651.

. G. Nielsen and T.G. Nielsen, 1994: Microscale patchiness of plankton within a sharp pycnocline. J. Plankton Res.. 16. $5+3-554$. 